\title{
Contributions of genetically-modified animal models to the understanding and intervention in autosomal dominant polycystic kidney disease: present and future
}

\author{
Andressa Godoy Amaral' ${ }^{1}$, Elieser Hitoshi Watanabe ${ }^{2}$, Luiz Fernando Onuchic ${ }^{3}$
}

Amaral AG, Watanabe EH, Onuchic KLF. Contributions of gnetically-modified animal models to the understanding and intervention in autosomal dominant polycystic kidney disease: present and future. Rev Med (São Paulo). 2016 July-Aug.;95(Special Issue 2):10-21 .

\begin{abstract}
Autosomal dominant polycystic kidney disease (ADPKD) is the most prevalent monogenic renal disease, representing the fourth cause of end-stage kidney disease. This disorder occurs due to mutations in the PKD1 (Polycystic Kidney Disease 1) or $P K D 2$ (Polycystic Kidney Disease 2) genes, with most of the cases caused by mutations in $P K D 1$. The products of these genes, polycystin-1 (PC1) and polycystin-2 (PC2), are integral membrane glycoproteins that form a complex expressed in the surface of primary apical cilia of several cells, including renal tubular cells. Such proteins are also expressed in other subcellular sites. PC2 functions as a non-selective cation channel with high permeability to calcium, while $\mathrm{PC} 1$ is thought to function as a membrane receptor and likely as an adhesion molecule. Since the discovery and characterization of $P K D 1$ and $P K D 2$, the generation of genetically-modified animal models has prompted remarkable advances in the elucidation of ADPKD pathogenesis and identification of potential therapeutic targets. Such animals included knockout, knockin and spatial and temporal conditional knockout models. These progresses allowed the recognition of a complex
\end{abstract}

genetic network involved in the modulation of polycystic kidney disease and the identification of potential modifiers of ADPKD. The mentioned advances also allowed the performance of strategic preclinical studies in mouse models orthologous to this disease, creating appropriate platforms to support robust clinical trials. The generation of composed mutants will likely lead to progressively more complex and specific analyses of ADPKD pathogenesis in the next decades, with meaningful clinical consequences. Moreover, the technical complexity and speed of generating strategic genetically-modified animal models has dramatically improved in recent years, considerably expanding the possibilities for the coming future. In addition, recently developed in vitro approaches such as induced pluripotent stem cells, kidney-on-a-chip and kidney organoid technologies are thought to bring robust and complementary future inputs to the understanding and therapy directed to ADPKD.

Descritores: Animals, genetically modified; Kidney diseases/ genetics; Polycystic Kidney, Autosomal Dominant.

\section{INTRODUCTION}

$\mathrm{T}$ The expansion of molecular genetics knowledge in the last decades has dramatically modified the understanding of mechanisms of life and disease. It is worth to note that the obtained invaluable achievements in this field were brought to light by an audacious shift of the paradigm of scientific thinking that occurred in the beginning of the last century. Until then, the classic medical science was built based on observations of symptoms and tissues abnormalities. While this scientific approach resulted in significant medical advances, it was unable to

1. MS, University of São Paulo School of Medicine, Department of Medicine, Divisions of Nephrology and Molecular Medicine. Email: andressa_amaral@usp.br.

2. MD, University of São Paulo School of Medicine, Department of Medicine, Divisions of Nephrology and Molecular Medicine. Email: elieserhw@yahoo.com.

3. MD, PhD, University of São Paulo School of Medicine, Department of Medicine, Divisions of Nephrology and Molecular Medicine (corresponding author). Email: lonuchic@usp.br.

Mailing address: Luiz Fernando Onuchic. Av. Dr. Arnaldo, 455 - Sala 4304. 01246-903 São Paulo, SP, Brazil. 
establish deep, detailed disease mechanisms and causative links, providing poor substrate to understand pathogenesis and limited inputs to therapeutic development. In this context, major medical discoveries derived from fortuitous experimental findings or ancient traditional knowledge, including penicillin, early chemotherapy drugs and acetyl salicylic acid. Back then drug development was mainly dependent on insights gathered from the observation of natural events. Examples of this reality include the angiotensin-converting enzyme inhibitors, developed upon the recognition of the vasodilator effect determined by a serpent venom, and curare, derived from the observation of muscular palsy induced by a plant toxin.

In the 1930s, however, advances of genetics, microbiology, biochemistry and physics were brought together to allow the development of a new revolutionary investigative approach, molecular biology. This method anchors the understanding of the biological phenomena, starting from the characterization and study of macromolecules. The knowledge construction, therefore, started to be supported and generated upon solid scientific data, obtained from rigorous observation and/or controlled experimentation. This approach led to consolidation of robust platforms of normal and pathogenic molecular mechanisms that enabled the development of potentially accurate and non-toxic therapeutic interventions.

Interestingly, the initial molecular discoveries showed that the fundamental biological processes were widely conserved along the phylogenetic evolution. This high similarity with other species, in particular with superior eukaryotes, allied to ethical limitations of clinical studies and high complexity of biological phenomena, created the scenario and the need to develop cell and animal models of human disease. Since then animal models have provided critical contributions to research directed to human diseases. The remarkable advances in autosomal dominant polycystic kidney (ADPKD) constitute one of the histories that document this reality, bringing promising perspectives to the field.

ADPKD is the most common monogenic lifethreatening disease, with an estimated prevalence of 1:400-1000 in the general population ${ }^{1,2}$. This disease is characterized by progressive development of multiple renal cysts and a number of extrarenal manifestations. The renal phenotype includes a gradual replacement of renal tubules by cysts that arise from any nephron segment, although those derived from collecting ducts are usually larger and more numerous. This process is accompanied for many years by reasonably preserved renal function, followed by a period of relatively fast decline in glomerular filtration rate (GFR). As consequence, end-stage kidney disease (ESKD) develops in approximately half of the patients by the sixth decade ${ }^{3}$. The extrarenal manifestations, in turn, comprise 1) cardiovascular abnormalities, including mitral valve prolapse, intracranial and aortic aneurysms, and left ventricular hypertrophy; 2) gastrointestinal abnormalities, such as hepatic cysts with marked organ growth in some cases, pancreatic cysts, and colon diverticula; and 3) additional manifestations, including seminal vesicle and epididymal cysts, abdominal hernias and splenic cysts ${ }^{4,5}$.

The identification of genes mutated in ADPKD allowed the characterization of its products and the progressive elucidation of genetic, molecular and cellular mechanisms involved in this disorder's pathogenesis. The gene mutated in most ADPKD patients, PKD1 (polycystic kidney disease 1), was identified and partially characterized in 1994, and fully characterized in $1995^{6,7}$. This gene encodes a $14-\mathrm{kb}$ transcript and is related to six pseudogenes formed by repeated duplications on chromosome 16 . In 1996 Mochizuki et al identified the second ADPKD causative gene, PKD2 (polycystic kidney disease 2$)^{8}$. Depending on the nature of the analyzed population, 64$85 \%$ of the cases are linked to the PKD1 locus ${ }^{9}$. This form of the disease, referred as ADPKD1, is usually associated with a more severe phenotype than ADPKD2, caused by mutation in $P K D 2^{10}$.

PKD1 encodes polycystin-1 (PC1), a large membrane protein that likely functions as a cell surface receptor and as an adhesion molecule ${ }^{11}$. PKD2, in turn, encodes polycystin-2 (PC2), a member of the transient receptor protein polycystin (TRPP) family of calcium channel proteins ${ }^{12}$. The two gene products can interact intracellularly through coiled-coiled domains and are found co-assembled or alone in a variety of cellular locations. The interaction between $\mathrm{PC} 1$ and $\mathrm{PC} 2$ is best characterized in primary apical cilia (PAC) of kidney tubular epithelial cells. In this organelle, the complex PC1/PC2 appears to act as a sensor that regulates ciliary responses to mechanical 
stimulation, generating calcium influx ${ }^{13,14}$ (Figure 1). These proteins are involved in signaling transduction pathways essential for maintenance of differentiated, polarized, predominantly reabsorptive tubular epithelial phenotypes, as well as for modulation of proliferation and apoptosis ${ }^{15}$. Therefore, mutations in either PKD1 or PKD2 may disrupt these fundamental signaling pathways and the mentioned cellular features, giving rise to cells with an aberrant phenotype.

Interestingly, many of the genes involved in human cystic kidney diseases are expressed in PAC. The PAC, classified as non-motile cilium, is a sensory organelle that projects itself from the surface of most cells. This structure has a fundamental role in cellular signaling, including cell growth and differentiation, orientation of planar cell polarity and division, cell proliferation and apoptosis ${ }^{16}$.

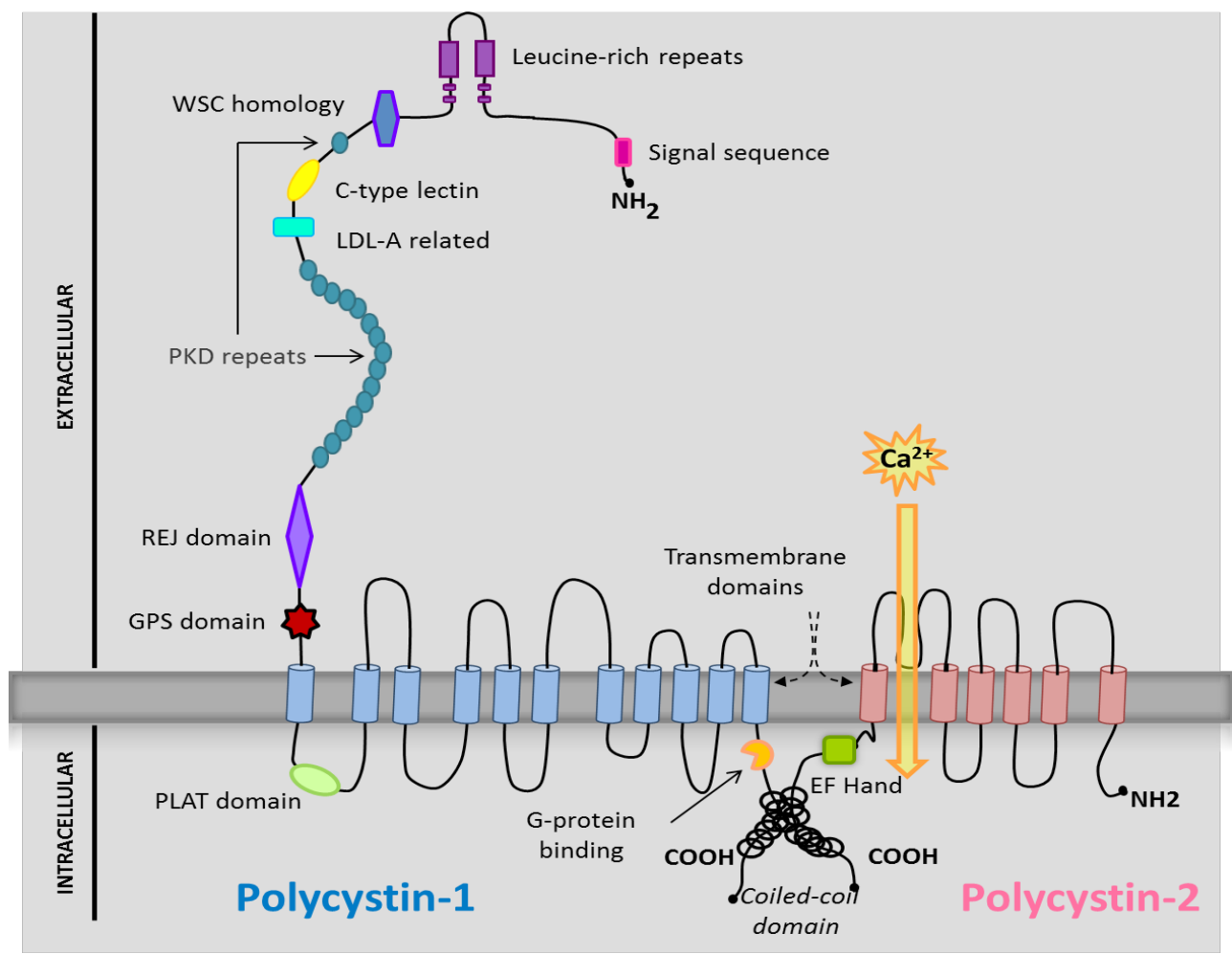

Figure 1: Polycystin-1 and polycystin-2 structure. The protein domains are identified in the figure. ER = endoplasmic reticulum; GPS $=$ G-protein-coupled receptor proteolytic site; LDL-A = low-density lipoprotein A; PLAT = polycystin-1, lipooxygenase, alpha toxin; $\mathrm{REJ}=$ receptor for egg-jelly; WSC $=$ cell-wall and stress-response component

Although all ADPKD tubular epithelial cells harbor a $P K D 1$ or $P K D 2$ germline mutation in one allele, renal cysts develop in less than $1 \%$ of the nephrons. The understanding of the focal nature of cyst formation in ADPKD came with the discovery that cystic lining cells of human kidneys and liver display a somatic mutation in the previously normal PKD1 or PKD2 allele ${ }^{17,18,19}$. Based on these findings, a 'two-hit' model was proposed, where the germline mutation constitutes the first hit, while the second hit is represented by the mentioned somatic mutation, which inactivates or significantly reduces the activity of previously normal gene copy. The mechanism of cyst formation in ADPKD, however, has been comprehensively analyzed in recent years, expanding the proposed two-hit model. As discussed ahead, this progress was achieved based on the generation of strategic genetically-modified mouse models.

\section{Generation of Genetically-Modified Mouse Models}

Despite major contributions brought by in vitro molecular models, many issues related to ADPKD pathogenesis could not be elucidated in this scenario. In vivo models expanded such possibilities, allowing more complex, deeper and functional investigation. Since mice share $99 \%$ of their genes and most of their physiological 
and pathological features with humans, mouse models orthologous to ADPKD are shedding new light on the mechanisms of this illness, in addition to providing the necessary platform for pre-clinical studies. In this review, therefore, we will focus on these genetically-engineered mouse models and their hallmark contributions to the understanding of ADPKD pathogenesis and the proposal of new potential therapeutic interventions.

Following the complete structural characterization of PKD1 and PKD2, mouse models were created with targeted mutations to the corresponding mouse orthologues, $P k d 1$ and $P k d 2$. Gene targeting is widely used to inactivate a gene (knockout), but it also can be used to insert point mutations, micro-deletions or exogenous DNA insertions at specific loci and genomic positions. This technique was anchored on the discovery of homologous recombination and the isolation of murine embryonic stem cells (ES cells $)^{20}$. This process begins with the construction of a vector containing the modified gene sequence, selection markers and homologous DNA flanking sequences. The targeting vector is then added to ES culture cells and, by homologous recombination, the engineered construct finds the targeted gene and recombination takes place within the homologous sequences. Positive and negative selection is used to enrich for ES cells that incorporated the modified DNA sequence in the correct position. Following appropriate selection, ES cells that incorporated the vector are clone-expanded in culture (Figure 2A). These ES cells are injected into blastocysts, which are implanted into foster mothers to generate chimeric mice able to transmit the mutant gene to their progeny. To facilitate isolation of the desired progeny, the ES cells and recipient blastocysts are derived from mice with different coat colors, associated with distinct alleles. Because of this cellular mixing, the resultant offspring is called chimeric; many of its cells are derived from the original blastocyst while part of its cells are derived from recombinant ES cells. If the gonads of the chimeric mice were derived from ES cells, its breeding with normal mouse will yield offsprings with the coat color corresponding to the ES cells and heterozygous for the modified gene (Figure 2B).
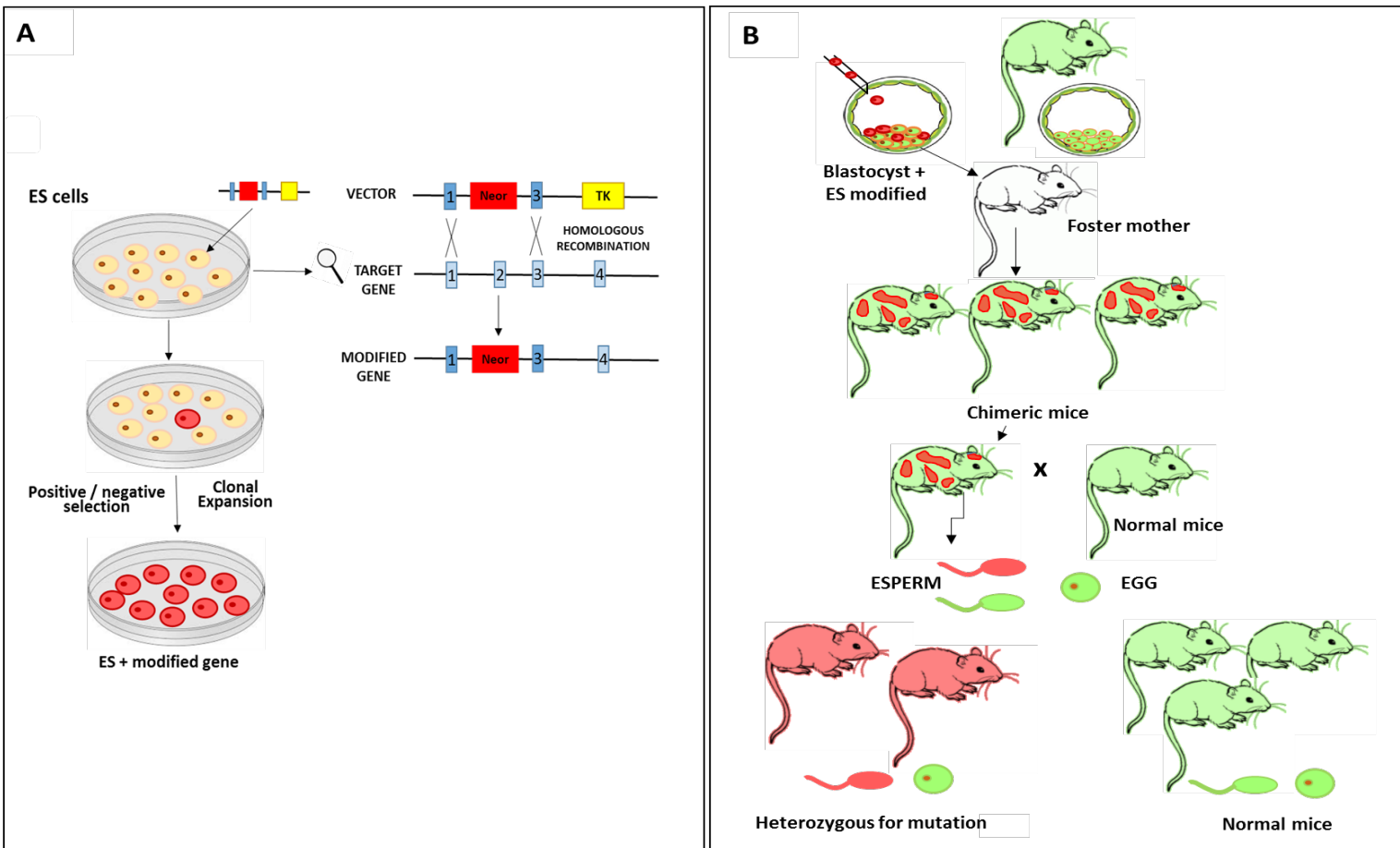

Figure 2: Generation of gene-targeted mice. A) Construction of targeting vector containing the modified gene sequence, selection markers and homologous DNA flanking sequences. The vector is added to ES culture and recombination takes place within the homologous sequences. Positive and negative selection is used to enrich for ES cells that incorporated the modified gene in the correct position; B) The modified ES cells are injected into blastocysts, which are implanted into foster mothers to generate chimeric mice able to transmit the mutant gene to their progeny. ES cells = embryonic stem cells, Neor = neomycin-resistance gene (positive selection marker), TK= thymidine kinase (negative selection marker) 
More recently, the conditional Cre-LoxP system has been used to inactivate or activate genes of interest in a spatial and temporal manner. Derived from the P1 bacteriophage, Cre is a site-specific DNA recombinase (Cre) that recognizes the 34-bp nucleotide sequence loxP (locus of $X$-over of $P 1$ ) and catalyzes both intra and intermolecular recombination between two loxP sites $^{21}$. Using homologous recombination technology, a targeting vector with loxP sites flanking a specific gene region can be incorporated. Once Cre is expressed, the region of DNA placed between two loxP sites will be excised. For conditional inactivation of a given gene, tissue-specific and inducible promoters can be used to drive Cre expression. Reporter Cre strains have been recently engineered to express a reporter gene following the removal of a loxP-flanked STOP cassette, marking cell lineages that can be targeted with a given Cre line. This methodology can be applied for directing lacZ or GFP driving the expression of the $\beta$-galactosidase and green fluorescent protein, respectively, allowing the identification of the cell recombination promoted by Cre activity.

Conditional knockout models are particularly important to study ADPKD due to phenotype specificities yielded by $P k d 1$ and $P k d 2$ inactivation. Along this line, the Jackson Laboratories currently offer more than 300 Cre transgenic mouse strains with different promoters and site of expression, bringing huge possibilities to study unresolved questions related to ADPKD pathogenesis.

\section{Orthologous Mouse Models: Contributions to Elucidation of ADPKD Pathogenesis and Pre-Clinical Studies}

\section{Cystogenesis}

In a short period of time, several $P k d l$ and $P k d 2$ mouse lines have been established with null or truncating mutations $\left(\mathrm{Pkd1}^{+/-} \text {and } \mathrm{Pkd2^{+/ }}\right)^{22-31}$. As expected, homozygous null mutant mice are embryonic lethal and develop severely cystic kidneys in utero. The heterozygotes, in turn, developed few cysts later in life, mainly in the liver. Further evidence comes from a mouse line carrying an unstable allele $\left(P k d 2^{\mathrm{wS} 25}\right)$ that is prone to genomic rearrangement and consequent generation of a null or wild-type allele ${ }^{29}$. Compound heterozygous
$P k d 2^{\mathrm{WS} 25 /-}$ mice, representing an authentic animal model of human ADPKD, are born live but develop severely polycystic kidneys during adulthood. Interestingly, PC1 expression was detected in tubules but not in the cyst epithelium of $P k d 1^{+/}$mice ${ }^{23,29}$, similarly to what has been found in humans ${ }^{17}$.

Despite strong supporting evidence, the two-hit gene mutation model does not sufficiently explain a number of observations in humans or mice with ADPKD. Hypomorphic homozygous mouse models have reduced $P k d 1$ or $P k d 2$ expression in all cells in which the gene is expressed, or carry a missense mutation that reduces protein expression and function ${ }^{32-35}$. These knockin models are perinatally viable and develop cystic phenotype. It must be pointed out that cyst formation may occur in a given cell when the PC1 or PC2 activity falls below a critical level, called "cystogenic threshold".

The tissue and/or time-specific conditional knockout models enable a well-controlled step-by-step analysis of gene inactivation and cyst formation. Using this breakthrough technology, studies revealed an important change in response to inactivation of both $P k d l$ copies depending on the life stage of the kidney ${ }^{26,36,37}$. Using a tamoxifen-inducible $P k d 1$ conditional knockout, Piontek et al. ${ }^{36}$ showed that when $P k d 1$ inactivation occurred before postnatal day 13 , the mice developed severely cystic kidneys, whereas inactivation after postnatal day 13 resulted in only late onset cysts. Independently, Lantingavan-Leeuwen et al. ${ }^{26}$ and Takakura et al. ${ }^{37}$, demonstrated that $P k d l$ inactivation in adult mice is not sufficient to induce massive cyst formation. On the other hand, $P k d 1$ inactivation in the first week of life resulted in severely cystic phenotype. Taken together, these data support the hypothesis that inactivation of $P k d l$ would not be sufficient to initiate the necessary cell proliferation for significant cyst formation in the adult kidney. These data led the investigators to propose that the rapid development of cysts in the mature kidney might require a third hit in addition to the inactivation of both $P k d 1$ alleles. A subsequent study from the same group was able to prove this point, showing that renal ischemia/reperfusion (IR) can act as a third hit for cyst formation in adult kidneys ${ }^{38}$. Mice submitted to $P k d 1$ inactivation at the age of 5 weeks and to unilateral renal IR at 8 weeks developed significantly cystic formation in 
the kidney submitted to injury, a phenotype not observed in the contralateral kidney not submitted to IR. In parallel, Bastos et al. ${ }^{39}$ also showed that $P k d 1^{+/}$mouse submitted to renal IR developed microcysts 6 weeks after the insult. In this context, IR likely represents an additional hit in mature kidneys with $P k d l$ inactivation, reactivating kidney developmental programs and/or promoting increased cell proliferation, which can create the cellular environment required for rapid and broad cyst formation.

These results have expanded and improved the two-hit model for cystogenesis. In this new scenario, cyst formation starts in a given cell when PC1 or PC2 activity falls below a critical level. This threshold, however, is believed to be strongly dependent on the developmental stage and the superimposition of environmental events such as renal injury.

Polycystin-1 cleavage and maturation

An important study showed that recombinant human PC1 is cis-autoproteolytically cleaved at the G proteincoupled receptor proteolytic site domain (GPS) which is located just before the beginning of the first transmembrane domain ${ }^{40}$. The cleavage results in two distinct products, the $\mathrm{N}$-terminal fragment (NTF) and the C-terminal fragment (CTF). To investigate this process in vivo, a $P k d l$-knockin mouse model $\left(P k d I^{\mathrm{v} / v}\right)$ was generated to suppress PC1 cleavage ${ }^{35}$. This hypomorphic allele was created by inserting the T3041V missense mutation, which modifies the GPS-mediated cis-autoproteolysis site without affecting the structure and function of the protein. The $P k d I^{\mathrm{V} / v}$ mice show a hypomorphic phenotype with lower body growth and progressive renal failure from the 8th postnatal day on. This phenotype evolves into a severe renal cystic involvement and intensely reduced survival, often less than 25 days. In conclusion, PC1 full length plays a critical role during embryonic development, whereas the GPS-cleaved PC1 products are essential for postnatal kidney maturation and maintenance ${ }^{35}$.

PC1 maturation depends on its cleavage and posttranslational modifications that results in a glycosylated PC1 N-terminal product (PC1-NTR). Interestingly, PC1/ PC2 interaction seems to be essential in this process. According to Chapin et al., PC2 expression is crucial to both
PC1 GPS cleavage and PC1 appearance at the plasma and ciliary membrane. Mutations that prevent GPS-dependent PC1 cleavage hinder its plasma membrane localization ${ }^{42}$. A later study showed that PC1 and PC2 initially interact in the ER before GPS cleavage and that this interaction is central to the subsequent PC1 maturation, including exiting from the endoplasmic reticulum and cell surface localization. In this process, $\mathrm{PC} 2$ acts as an essential chaperone for $\mathrm{PC} 1$ maturation $^{43}$. A hypomorphic mouse model ( $P k d l^{\mathrm{RC} /}$ $\left.{ }^{\mathrm{RC}}\right)$ that develops gradual cystogenesis displays reduced levels of PC1-NTR in renal tissue and urinary exosomelike vesicles ${ }^{34}$, supporting an essential role of mature PC1 in kidney maintenance.

\section{Cardiovascular dysfunction in ADPKD}

Several studies have demonstrated that cardiovascular alterations seem to initiate early in the course of ADPKD and constitute a major cause of morbidity and mortality in this disease ${ }^{44,45}$. In this context, systemic arterial hypertension (SAH) is present in about $60 \%$ of patients with ADPKD prior to significant renal dysfunction ${ }^{46,47}$ and 10 years earlier than in the general population ${ }^{48}$. The ADPKD cardiovascular phenotype also includes increased left ventricular mass ${ }^{49}$ and idiopathic dilated cardiomyopathy ${ }^{50}$.

In order to test whether cystic disease is the principal determinant of hypertension, Fonseca et al investigated blood pressure-related phenotypes in two independent mouse models orthologous to ADPKD1. The authors found that $P k d 1^{+/}$(HT) mice, a non-cystic mouse at the analyzed age, were normotensive whereas $P k d l^{\text {cond/ }}$ cond:Nestin ${ }^{\text {cre }}$, cystic animals with a mosaic pattern of gene inactivation (CY), were hypertensive at the ages of 5 and 10-13 weeks and showed increased kidney expression of renin-angiotensin system (RAS) components. Given the complementary nature of the evaluated $P k d l$-deficient models, these results provide key insights into the understanding of development of hypertension in ADPKD. These data support the concept that distension of cysts leads to sectorial vascular compression and consequent focal areas of renal ischemia, leading to RAS activation and hypertension ${ }^{51}$. Using the same mouse models, a more recent study performed in our laboratory revealed decreased 
myocardial deformation and systolic function in CY at 5-6 and 20-24 weeks (wk) of age and in HT noncystic mice at 5-6 and 10-13 wk, compared to their controls. CYG+ and $\mathrm{HTG}+$ hearts presented reduced $\mathrm{PC} 1$ expression, increased apoptosis and mild fibrosis ${ }^{52}$. Our findings demonstrate an important myocardial dysfunction in different $P k d 1$ deficient models suggesting a pivotal role of PC1 in the heart.

Genetic interaction in ADPKD and modifier loci

ADPKD represents an imbalance of a complex network of genetic interactions. Unraveling such interactions is fundamental to understand this disease pathogenesis. To address this question, Garcia-Gonzalez et al generated a mouse model of autosomal recessive polycystic kidney disease (ARPKD) that closely reproduced the renal human phenotype. This animal is homozygous for a hypomorphic Pkhd1 allele - the mouse orthologue to PKHD1, the gene mutated in human ARPKD. When combined with $P k d 1$ haploinsufficiency, these mice displayed significant worsening of the kidney cystic phenotype, indicating genetic interaction between $P k d 1$ and $P k h d 1^{53}$.

Another key study utilized mice with altered expression of genes associated with ADPKD ( $P k d 1$ and $P k d 2$ ), ARPKD (Pkhdl) and autosomal dominant polycystic liver disease $(\operatorname{Sec} 63 \text { and Prkcsh })^{54}$. In this case, decreased activity of $P k d 1$ or $P k d 2$, associated with reduced function of the other genes worsened the cystic phenotype. In contrary, $P k d 1$ overexpression, but not $P k d 2$, significantly attenuated the cystic burden. These data supported the concept of a genetic network, including these five genes, and place $P k d 1$ deficiency as the critical player in cystogenesis.

The PAC subcellular localization of the complex $\mathrm{PC} 1 / \mathrm{PC} 2$ is critical in cyst formation and growth. Their specific roles in this organelle, however, remain poorly understood. To address this question, Ming et al combined highly cystic models deficient in Pkd1 or Pkd2 with Ift20 or Kif3a-deficient mice, creating strategic double-mutant animals ${ }^{55}$. The two last genes are required for maintenance of PAC integrity, and the lack of their function typically leads to a mildly cystic phenotype in mice. Interestingly, the PAC loss in the double mutants resulted in intermediate polycystic kidney severity. The severity of the renal phenotype was more intense for longer time intervals between the losses of polycystins and PAC. This principle was verified for developmental and mature kidneys. These data indicate that an intact PAC in required for rapid cyst growth and support a regulatory role of polycystins in this organelle.

As previously mentioned, we have shown that $P k d 1$ deficient mice develop cardiac dysfunction ${ }^{52}$. In the same study, double-mutant mice were generated to investigate galectin-3 as a potential modifier of the ADPKD cardiac phenotype. When Pkd1-deficient mice were placed into a homozygous Lgals3 (the gene that encodes galectin-3) null allele background, their cardiac phenotype was largely rescued. Taken together, our findings demonstrated genetic interaction between Pkdland Lgals3 and revealed the potential of galectin-3 as a biomarker and as a target for future therapies directed towards heart dysfunction in this disease ${ }^{52}$.

Efforts directed to modifier loci have recently included a comprehensive network analysis of gene expression, aiming to find a specific signature of ADPKD. Using this approach, Menezes et al compared $P k d 1$ deficient mice with their normal controls and found quite similar expression patterns between the groups between post-natal days 12 and 14. The network, however, was enriched by metabolic pathways in both groups, suggesting that the metabolic state may be an important determinant of disease severity ${ }^{56}$.

Animal models in translational medicine as platforms for disease elucidation and clinical trials

Based on whole exome analyses from patients with genetically unclear renal cystic diseases, a number of new genes have been associated to these disorders. Interestingly, the genes FAN1, MRE11, ZNF423, and CEP164, recently associated to nephronophthisis-related ciliopathies, are involved in DNA damage repair ${ }^{57}$. Key mutations identified in humans may provide valuable insights to unravel pathophysiologic mechanisms when reproduced in animal models. The generation of a strategic mouse line including the human $P K D 1$ hypomorphic variant p.R3277C is a good example of such an approach ${ }^{34}$. Homozygotes for this allele 
develop a slowly progressive disease that resembles the human illness progression, a feature that makes this model appropriate for preclinical research.

The recent development of induced pluripotent stem cell (iPSC) protocols, in turn, expands the applications of animal models. iPSCs are created by genetic reprograming somatic cells, allowing investigation of diseases based on the generation of specifically-diseased cell lines derived from humans. This technology has the advantage of transposing the patient genetic background to the research setting. Combined with animal models, iPSCs and/or their products may provide comprehensive platforms for robust and integrated disease research.

Animal models have become essential to analyze pathways altered in cyst-lining cells. This information has provided potential targets for therapeutic interventions. Reduced activity of the polycystin complex leads to defective homeostasis of intracellular calcium $\left(\mathrm{Ca}^{2+}\right)$ in ADPKD cells. Low intra-cytosolic $\mathrm{Ca}^{2+}$ levels stimulate adenylyl cyclase 6 and inhibits phosphodiesterases 1 and 3 , favoring intracellular generation and accumulation of cAMP (58). In contrast to normal kidney tubular cells, the intracellular accumulation of cAMP activates the mitogen-activated protein kinase/ERK (MAPK/ERK) pathway, promoting cell proliferation and fluid secretion, processes that participate in cyst growth ${ }^{59,60}$. In fact, decreased intracellular cAMP induced by a vasopressin V2receptor antagonist ameliorated cystic disease progression in orthologous and non-orthologous ADPKD murine models $^{61-63}$. On the basis of results obtained in animal models, a clinical trial (TEMPO 3:4) was performed in ADPKD patients using tolvaptan, a vasopressin V2receptor antagonist. Notably, treatment with tolvaptan slowed the rate of total kidney volume (TKV) growth and the rate of estimated GFR (eGFR) decline ${ }^{64}$. Recently, the European Medicines Agency approved the use tolvaptan to slow the progression of $\mathrm{ADPKD}^{65}$.

Somatostatin is a peptide inhibitory hormone that signals via somatostatin receptors to inhibit the generation of cAMP. In this context, a recent trial with the long-acting somatostatin analog octreotide revealed reduction in TKV growth rate in the treated patients compared to the placebo group after one year, however significance was no longer detected after three years ${ }^{66}$. These findings provide a background for large trials to test the renal protective effect of somatostatin analogues in ADPKD.

Since hypertension is a major manifestation in ADPKD, with heavy impact on cardiovascular morbidity and mortality, and is associated with accelerated progression to ESKD, large clinical trials - HALT-PKD study- $\mathrm{A}^{67}$ and $\mathrm{B}^{68}$ - were designed to assess potential benefits of rigorous blood pressure control and inhibition of RAS in ADPKD. Study-A analyzed patients with early renal disease, whereas study-B analyzed patients with late disease. This study revealed that patients with eGFR $>60$ $\mathrm{mL} / \mathrm{min} / 1.73 \mathrm{~m}^{2}$ and rigorous BP control (target of 95 110/60-75-mmHg) displayed a lower annual rate of TKV growth, left-ventricular-mass index and albuminuria than patients within the same range of renal function submitted to conventional BP control (120-130/70-80-mmHg target). However, no significant difference in the rate of eGFR decline was detected. In addition, combined therapy with angiotensin-converting enzyme inhibitor (ACEI) and angiotensin receptor blockage showed no renal benefit over ACEI alone both for patients with eGFR $>60$ and between $25-60 \mathrm{~mL} / \mathrm{min} / 1.73 \mathrm{~m}^{2}$.

The mTOR (mammalian target of rapamycin) signaling pathway is essential for cell growth, proliferation and survival. This pathway is upregulated in cyst-lining cells both in patients and in animal models of ADPKD. In several pre-clinical studies, mTOR inhibitors ameliorated cystic disease progression ${ }^{69-72}$. Based on this knowledge, two independent large clinical trials were performed in ADPKD patients using mTOR inhibitors ${ }^{73,74}$. Such trials targeted patients within different ranges of renal function. Disappointing results were obtained, however, as mTOR inhibition did not show significant effects on disease progression in both studies. Dose limiting due to side effects may have contributed to the lack of clinical success.

As observed, biological differences between humans and rodents and the complexity of the signaling-pathway network related to ADPKD are significant challenges to overcome in translating data and information from the preclinical to the clinical reality. Combined treatment with a number of agents, however, may become an effective therapy in the future, since this approach could be potentially applied using lower doses, avoiding unwanted 
adverse effects, while promoting additive beneficial effects.

\section{Future technologies and perspectives}

Possibilities of genetic engineering have become more efficient and cost-effective. Notably, the CRISPR/ Cas-9 platform allows the generation of specific knockout mice in six months ${ }^{75}$. This system uses an artificial guide RNA complementary to the genomic target sequence conjugated to a bacterial protein capable of activating caspase 9, therefore leading to DNA cleavage. The subsequent DNA non-homologous repair often leads to small deletions or inclusions, resulting in loss of gene function.

Future perspectives include the generation of kidney organoids in 3D cultures, derived from human pluripotent stem cells. These cells are submitted to systematic differentiation, giving rise to integrated cell populations with features of proximal tubule, podocytes and endothelium, constituting the organoid. This complex structure can reproduce some absorptive and post-injury response patterns observed in proximal tubule ${ }^{76}$. Another recently developed technology directed to elucidate mechanisms and evaluate potential therapies is the kidney-on-a-chip platform ${ }^{77}$. This modern device allows functional assessment of appropriately-organized cell preparations reproducing the basic features of the kidney. These approaches, however, are still far from reproducing the complexity of in vivo models, emphasizing the pivotal

\section{REFERENCES}

1. Harris PC, Torres VE. Polycystic kidney disease. Annu Rev Med. 2009;60:321-37.

2. Lee SH, Somlo S. Cyst growth, polycystins, and primary cilia in autosomal dominant polycystic kidney disease. Kidney Res Clin Pract. 2014;33(2):73-8.

3. Reed BY, McFann K, Bekheirnia MR, Reza Bekheirnia M, Nobakhthaghighi N, Nobkhthaghighi N, et al. Variation in age at ESRD in autosomal dominant polycystic kidney disease. Am J Kidney Dis. 2008;51(2):173-83.

4. Gabow PA. Autosomal dominant polycystic kidney disease. N Engl J Med. 1993;329(5):332-42.

5. PKD Fountation 2015. Available from: http://www.pkdcure. org/.

6. The European Polycystic Kidney Disease C. The polycystic contributions and need of animals models for the next decades.

Despite the relevant knowledge accumulation in ADPKD molecular pathogenesis, the genetic interactions and cellular pathways are still largely unknown. The current data suggest $P K D 1$ and $P K D 2$ participation in dynamic and integrative ways, varying according to the cellular and systemic status. Modern technologies allow control of the time, tissue and pattern of gene inactivation in a relatively fast and available fashion. This setting facilitates the generation of animals carrying multiple geneticallyengineered alleles, enabling the investigation of genetic interactions in different stages of development, in kidney injury, and in other strategic conditions. This approach may be allied to the use of modern reporter genes, allowing more precise tracking of cellular recombination events. The application of network expression platforms, in turn, has the potential of returning a comprehensive view and critical analysis of alterations in the cell pathway network. Such possibilities are likely to favor the identification of ADPKD modifier loci and new potential therapeutic targets.

Recently developed in vitro approaches such as the kidney-on-a-chip and kidney organoid technologies may also provide additional robust and complementary inputs to the comprehension and treatment of ADPKD. Along these lines, in the next decades the scientific community and patients will likely experience a dramatic improvement in ADPKD understanding and intervention, provided by the successive steps of molecular and cellular translational medicine.

kidney disease 1 gene encodes a $14 \mathrm{~kb}$ transcript and lies within a duplicated region on chromosome 16. Cell. 77(6):881-94

7. Polycystic kidney disease: the complete structure of the PKD1 gene and its protein. The International Polycystic Kidney Disease Consortium. Cell. 1995;81(2):289-98.

8. Mochizuki T, Wu G, Hayashi T, Xenophontos SL, Veldhuisen $\mathrm{B}$, Saris JJ, et al. PKD2, a gene for polycystic kidney disease that encodes an integral membrane protein. Science. 1996;272(5266):1339-42.

9. Robinson C, Hiemstra TF, Spencer D, Waller S, Daboo L, Karet Frankl FE, et al. Clinical utility of PKD2 mutation testing in a polycystic kidney disease cohort attending a specialist nephrology out-patient clinic. BMC Nephrol. 2012;13:79 
10. Hateboer N, v Dijk MA, Bogdanova N, Coto E, SaggarMalik AK, San Millan JL, et al. Comparison of phenotypes of polycystic kidney disease types 1 and 2. European PKD1PKD2 Study Group. Lancet. 1999;353(9147):103-7.

11. Sandford R, Mulroy S, Foggensteiner L. The polycystins: a novel class of membrane-associated proteins involved in renal cystic disease. Cell Mol Life Sci. 1999;56(7-8):567-79.

12. Tsiokas L, Arnould T, Zhu C, Kim E, Walz G, Sukhatme VP. Specific association of the gene product of PKD2 with the TRPC1 channel. Proc Natl Acad Sci U S A. 1999;96(7):39349.

13. Nauli SM, Alenghat FJ, Luo Y, Williams E, Vassilev P, Li X, et al. Polycystins 1 and 2 mediate mechanosensation in the primary cilium of kidney cells. Nat Genet. 2003;33(2):12937.

14. Wang S, Zhang J, Nauli SM, Li X, Starremans PG, Luo $\mathrm{Y}$, et al. Fibrocystin/polyductin, found in the same protein complex with polycystin-2, regulates calcium responses in kidney epithelia. Mol Cell Biol. 2007;27(8):3241-52.

15. Bastos AP, Onuchic LF. Molecular and cellular pathogenesis of autosomal dominant polycystic kidney disease. Braz J Med Biol Res. 2011;44(7):606-17.

16. Quinlan RJ, Tobin JL, Beales PL. Modeling ciliopathies: Primary cilia in development and disease. Curr Top Dev Biol. 2008;84:249-310.

17. Qian F, Watnick TJ, Onuchic LF, Germino GG. The molecular basis of focal cyst formation in human autosomal dominant polycystic kidney disease type I. Cell. 1996;87(6):979-87.

18. Pei Y, Watnick T, He N, Wang K, Liang Y, Parfrey P, et al. Somatic PKD2 mutations in individual kidney and liver cysts support a "two-hit" model of cystogenesis in type 2 autosomal dominant polycystic kidney disease. J Am Soc Nephrol. 1999;10(7):1524-9.

19. Watnick TJ, Torres VE, Gandolph MA, Qian F, Onuchic LF, Klinger KW, et al. Somatic mutation in individual liver cysts supports a two-hit model of cystogenesis in autosomal dominant polycystic kidney disease. Mol Cell. $1998 ; 2(2): 247-51$.

20. Dijkstra CD, Walvoort HC. The Nobel Prize in Physiology or Medicine 2007 for the development of 'knockout' technology. Ned Tijdschr Geneeskd. 2007;151(52):2875-6.

21. Sauer B, Henderson N. Site-specific DNA recombination in mammalian cells by the Cre recombinase of bacteriophage P1. Proc Natl Acad Sci U S A. 1988;85(14):5166-70.

22. Lu W, Peissel B, Babakhanlou H, Pavlova A, Geng L, Fan $\mathrm{X}$, et al. Perinatal lethality with kidney and pancreas defects in mice with a targetted Pkd1 mutation. Nat Genet. 1997;17(2):179-81.

23. Lu W, Fan X, Basora N, Babakhanlou H, Law T, Rifai N, et al. Late onset of renal and hepatic cysts in Pkd1-targeted heterozygotes. Nat Genet. 1999;21(2):160-1.

24. Kim K, Drummond I, Ibraghimov-Beskrovnaya O, Klinger $\mathrm{K}$, Arnaout MA. Polycystin 1 is required for the structural integrity of blood vessels. Proc Natl Acad Sci U S A. 2000;97(4):1731-6

25. Boulter C, Mulroy S, Webb S, Fleming S, Brindle K, Sandford R. Cardiovascular, skeletal, and renal defects in mice with a targeted disruption of the Pkd1 gene. Proc Natl Acad Sci U S A. 2001;98(21):12174-9.

26. Lantinga-van Leeuwen IS, Leonhard WN, van der Wal A, Breuning MH, de Heer E, Peters DJ. Kidney-specific inactivation of the Pkd1 gene induces rapid cyst formation in developing kidneys and a slow onset of disease in adult mice. Hum Mol Genet. 2007;16(24):3188-96.

27. Lu W, Shen X, Pavlova A, Lakkis M, Ward CJ, Pritchard L, et al. Comparison of Pkd1-targeted mutants reveals that loss of polycystin-1 causes cystogenesis and bone defects. Hum Mol Genet. 2001;10(21):2385-96.

28. Piontek KB, Huso DL, Grinberg A, Liu L, Bedja D, Zhao $\mathrm{H}$, et al. A functional floxed allele of Pkd1 that can be conditionally inactivated in vivo. J Am Soc Nephrol. 2004;15(12):3035-43.

29. Wu G, D’Agati V, Cai Y, Markowitz G, Park JH, Reynolds $\mathrm{DM}$, et al. Somatic inactivation of $\mathrm{Pkd} 2$ results in polycystic kidney disease. Cell. 1998;93(2):177-88.

30. Pennekamp P, Karcher C, Fischer A, Schweickert A, Skryabin B, Horst J, et al. The ion channel polycystin-2 is required for left-right axis determination in mice. Curr Biol. 2002;12(11):938-43.

31. Kim I, Ding T, Fu Y, Li C, Cui L, Li A, et al. Conditional mutation of Pkd2 causes cystogenesis and upregulates betacatenin. J Am Soc Nephrol. 2009;20(12):2556-69.

32. Lantinga-van Leeuwen IS, Dauwerse JG, Baelde HJ, Leonhard WN, van de Wal A, Ward CJ, et al. Lowering of $\mathrm{Pkd} 1$ expression is sufficient to cause polycystic kidney disease. Hum Mol Genet. 2004;13(24):3069-77.

33. Jiang ST, Chiou YY, Wang E, Lin HK, Lin YT, Chi YC, et al. Defining a link with autosomal-dominant polycystic kidney disease in mice with congenitally low expression of Pkd1. Am J Pathol. 2006;168(1):205-20.

34. Hopp K, Ward CJ, Hommerding CJ, Nasr SH, Tuan HF, Gainullin VG, et al. Functional polycystin-1 dosage governs autosomal dominant polycystic kidney disease severity. J Clin Invest. 2012;122(11):4257-73.

35. Yu S, Hackmann K, Gao J, He X, Piontek K, García-González MA, et al. Essential role of cleavage of Polycystin-1 at G protein-coupled receptor proteolytic site for kidney tubular structure. Proc Natl Acad Sci U S A. 2007;104(47):18688-93.

36. Piontek K, Menezes LF, Garcia-Gonzalez MA, Huso DL, Germino GG. A critical developmental switch defines the kinetics of kidney cyst formation after loss of Pkd1. Nat Med. 2007;13(12):1490-5.

37. Takakura A, Contrino L, Beck AW, Zhou J. Pkd1 inactivation induced in adulthood produces focal cystic disease. J Am Soc Nephrol. 2008;19(12):2351-63.

38. Takakura A, Contrino L, Zhou X, Bonventre JV, Sun Y, 
Humphreys BD, et al. Renal injury is a third hit promoting rapid development of adult polycystic kidney disease. Hum Mol Genet. 2009;18(14):2523-31.

39. Bastos AP, Piontek K, Silva AM, Martini D, Menezes LF, Fonseca JM, et al. Pkd1 haploinsufficiency increases renal damage and induces microcyst formation following ischemia/reperfusion. J Am Soc Nephrol. 2009;20(11):2389402.

40. Qian F, Boletta A, Bhunia AK, Xu H, Liu L, Ahrabi AK, et al. Cleavage of polycystin-1 requires the receptor for egg jelly domain and is disrupted by human autosomal-dominant polycystic kidney disease 1-associated mutations. Proc Natl Acad Sci U S A. 2002;99(26):16981-6.

41. Wei W, Hackmann K, Xu H, Germino G, Qian F. Characterization of cis-autoproteolysis of polycystin-1, the product of human polycystic kidney disease 1 gene. J Biol Chem. 2007;282(30):21729-37.

42. Chapin HC, Rajendran V, Caplan MJ. Polycystin-1 surface localization is stimulated by polycystin- 2 and cleavage at the $\mathrm{G}$ protein-coupled receptor proteolytic site. Mol Biol Cell. 2010;21(24):4338-48.

43. Gainullin VG, Hopp K, Ward CJ, Hommerding CJ, Harris PC. Polycystin-1 maturation requires polycystin-2 in a dosedependent manner. J Clin Invest. 2015;125(2):607-20.

44. Perrone RD, Ruthazer R, Terrin NC. Survival after endstage renal disease in autosomal dominant polycystic kidney disease: contribution of extrarenal complications to mortality. Am J Kidney Dis. 2001;38(4):777-84.

45. Ecder T, Schrier RW. Cardiovascular abnormalities in autosomal-dominant polycystic kidney disease. Nat Rev Nephrol. 2009;5(4):221-8.

46. Chapman AB, Schrier RW. Pathogenesis of hypertension in autosomal dominant polycystic kidney disease. Semin Nephrol. 1991;11(6):653-60.

47. Ecder T, Schrier RW. Hypertension in autosomal-dominant polycystic kidney disease: early occurrence and unique aspects. J Am Soc Nephrol. 2001;12(1):194-200.

48. Kelleher CL, McFann KK, Johnson AM, Schrier RW. Characteristics of hypertension in young adults with autosomal dominant polycystic kidney disease compared with the general U.S. population. Am J Hypertens. 2004;17(11 Pt 1):1029-34.

49. Chapman AB, Stepniakowski K, Rahbari-Oskoui F. Hypertension in autosomal dominant polycystic kidney disease. Adv Chronic Kidney Dis. 2010;17(2):153-63.

50. Paavola J, Schliffke S, Rossetti S, Kuo IY, Yuan S, Sun Z, et al. Polycystin-2 mutations lead to impaired calcium cycling in the heart and predispose to dilated cardiomyopathy. J Mol Cell Cardiol. 2013;58:199-208.

51. Fonseca JM, Bastos AP, Amaral AG, Sousa MF, Souza LE, Malheiros DM, et al. Renal cyst growth is the main determinant for hypertension and concentrating deficit in Pkd1-deficient mice. Kidney Int. 2014;85(5):1137-50.
52. Balbo BEP. Camundongos com deficiência em $P k d 1$ apresentam disfunção cardíaca, fenótipo atenuado por knockout de galectina-3. São Paulo: Universidade de São Paulo, Faculdade de Medicina; 2014.

53. Garcia-Gonzalez MA, Menezes LF, Piontek KB, Kaimori J, Huso DL, Watnick T, et al. Genetic interaction studies link autosomal dominant and recessive polycystic kidney disease in a common pathway. Hum Mol Genet. 2007;16(16):194050 .

54. Fedeles SV, Tian X, Gallagher AR, Mitobe M, Nishio S, Lee $\mathrm{SH}$, et al. A genetic interaction network of five genes for human polycystic kidney and liver diseases defines polycystin-1 as the central determinant of cyst formation. Nat Genet. 2011;43(7):639-47.

55. Ma M, Tian X, Igarashi P, Pazour GJ, Somlo S. Loss of cilia suppresses cyst growth in genetic models of autosomal dominant polycystic kidney disease. Nat Genet. 2013;45(9):1004-12.

56. Menezes LF, Zhou F, Patterson AD, Piontek KB, Krausz KW, Gonzalez FJ, et al. Network analysis of a Pkd1-mouse model of autosomal dominant polycystic kidney disease identifies HNF $4 \alpha$ as a disease modifier. PLoS Genet. 2012;8(11):e1003053.

57. Antignac C, Calvet JP, Germino GG, Grantham JJ, GuayWoodford LM, Harris PC, et al. The Future of Polycystic Kidney Disease Research--As Seen By the 12 Kaplan Awardees. J Am Soc Nephrol. 2015;26(9):2081-95.

58. Ye H, Wang X, Sussman CR, Hopp K, Irazabal MV, Bakeberg $\mathrm{JL}$, et al. Modulation of Polycystic Kidney Disease Severity by Phosphodiesterase 1 and 3 Subfamilies. J Am Soc Nephrol. 2016;27(5):1312-20.

59. Yamaguchi T, Hempson SJ, Reif GA, Hedge AM, Wallace DP. Calcium restores a normal proliferation phenotype in human polycystic kidney disease epithelial cells. J Am Soc Nephrol. 2006;17(1):178-87.

60. Li H, Findlay IA, Sheppard DN. The relationship between cell proliferation, $\mathrm{Cl}$ - secretion, and renal cyst growth: a study using CFTR inhibitors. Kidney Int. 2004;66(5):1926-38.

61. Gattone VH, Wang X, Harris PC, Torres VE. Inhibition of renal cystic disease development and progression by a vasopressin V2 receptor antagonist. Nat Med. 2003;9(10):1323-6.

62. Torres VE, Wang X, Qian Q, Somlo S, Harris PC, Gattone VH. Effective treatment of an orthologous model of autosomal dominant polycystic kidney disease. Nat Med. 2004;10(4):363-4.

63. Wang X, Gattone V, Harris PC, Torres VE. Effectiveness of vasopressin V2 receptor antagonists OPC-31260 and OPC41061 on polycystic kidney disease development in the PCK rat. J Am Soc Nephrol. 2005;16(4):846-51.

64. Torres VE, Chapman AB, Devuyst O, Gansevoort RT, Grantham JJ, Higashihara E, et al. Tolvaptan in patients with autosomal dominant polycystic kidney disease. N Engl J Med. 2012;367(25):2407-18. 
65. Gansevoort RT, Arici M, Benzing T, Birn H, Capasso G, Covic A, et al. Recommendations for the use of tolvaptan in autosomal dominant polycystic kidney disease: a position statement on behalf of the ERA-EDTA Working Groups on Inherited Kidney Disorders and European Renal Best Practice. Nephrol Dial Transplant. 2016;31(3):337-48.

66. Caroli A, Perico N, Perna A, Antiga L, Brambilla P, Pisani A, et al. Effect of longacting somatostatin analogue on kidney and cyst growth in autosomal dominant polycystic kidney disease (ALADIN): a randomised, placebo-controlled, multicentre trial. Lancet. 2013;382(9903):1485-95.

67. Schrier RW. Blood pressure in early autosomal dominant polycystic kidney disease. N Engl J Med. 2015;372(10):976-7.

68. Torres VE, Abebe KZ, Chapman AB, Schrier RW, Braun WE, Steinman TI, et al. Angiotensin blockade in late autosomal dominant polycystic kidney disease. N Engl J Med. 2014;371(24):2267-76.

69. Shillingford JM, Piontek KB, Germino GG, Weimbs T. Rapamycin ameliorates PKD resulting from conditional inactivation of Pkd1. J Am Soc Nephrol. 2010;21(3):489-97.

70. Wahl PR, Serra AL, Le Hir M, Molle KD, Hall MN, Wüthrich RP. Inhibition of mTOR with sirolimus slows disease progression in Han:SPRD rats with autosomal dominant polycystic kidney disease (ADPKD). Nephrol Dial Transplant. 2006;21(3):598-604.

71. Zafar I, Ravichandran K, Belibi FA, Doctor RB, Edelstein
CL. Sirolimus attenuates disease progression in an orthologous mouse model of human autosomal dominant polycystic kidney disease. Kidney Int. 2010;78(8):754-61.

72. Spirli C, Okolicsanyi S, Fiorotto R, Fabris L, Cadamuro M, Lecchi S, et al. Mammalian target of rapamycin regulates vascular endothelial growth factor-dependent liver cyst growth in polycystin-2-defective mice. Hepatology. 2010;51(5):1778-88.

73. Serra AL, Poster D, Kistler AD, Krauer F, Raina S, Young J, et al. Sirolimus and kidney growth in autosomal dominant polycystic kidney disease. N Engl J Med. 2010;363(9):820-9.

74. Walz G, Budde K, Mannaa M, Nürnberger J, Wanner C, Sommerer C, et al. Everolimus in patients with autosomal dominant polycystic kidney disease. N Engl J Med. 2010;363(9):830-40.

75. Sander JD, Joung JK. CRISPR-Cas systems for editing, regulating and targeting genomes. Nat Biotechnol. 2014;32(4):347-55.

76. Freedman BS, Brooks CR, Lam AQ, Fu H, Morizane R, Agrawal V, et al. Modelling kidney disease with CRISPRmutant kidney organoids derived from human pluripotent epiblast spheroids. Nat Commun. 2015;6:8715.

77. Wilmer MJ, Ng CP, Lanz HL, Vulto P, Suter-Dick L, Masereeuw R. Kidney-on-a-Chip Technology for DrugInduced Nephrotoxicity Screening. Trends Biotechnol. 2016;34(2):156-70. 